\title{
Erratum to: How to choose the best journal for your case report
}

Richard A. Rison ${ }^{1,2^{*}}$, Jennifer Kelly Shepphird ${ }^{3}$ and Michael R. Kidd ${ }^{4,5}$

\section{Erratum}

In the publication of this article [1], there are two errors in Table 1 Case report journals.

1. The error: 'The journal title BMJ Case Reports has no value in the PubMed Indexed column' Should instead read: 'BMJ Case Reports in the PubMed Indexed column should have indicated "Yes" because it is PubMed indexed"

2. The error: 'The journal title BMJ Case Reports has value "No" in the Open access column" Should instead read: "BMJ Case Reports in the Open access column should have indicated "Optional" because it does have an option for open access for an extra fee

This has now been included in this erratum.

\footnotetext{
* Correspondence: rison@usc.edu

${ }^{1}$ University of Southern California Keck School of Medicine, Los Angeles

County Medical Center, 12401 Washington Blvd, Whittier, CA 90602, USA

${ }^{2} \mathrm{PIH}$ Health Hospital-Whittier Stroke Center, PIH Health Hospital Non-Invasive

Vascular Laboratory, 12401 Washington Blvd, Whittier, CA 90602, USA
} 
Table 1 Case report journals

\begin{tabular}{|c|c|c|c|c|}
\hline Journal title & Publisher/Society & Year launched & Open access & PubMed indexed \\
\hline A\&A Case Reports & $\begin{array}{l}\text { Wolters Kluwer Health/International Anesthesia } \\
\text { Research Society }\end{array}$ & 2013 & No & No \\
\hline AACE Clinical Case Reports & American Association of Clinical Endocrinologists & 2015 & Yes & No \\
\hline ACG Case Reports Journal & American College of Gastroenterology & 2013 & Yes & Yes \\
\hline AJP Reports & Thieme Medical Publishers & 2011 & Yes & No \\
\hline American Journal of Cancer Case Reports & Ivy Union Publishing & 2013 & Yes & No \\
\hline American Journal of Case Reports & International Scientific Information & 2001 & Yes & Yes \\
\hline Aperito Journal of Case Reports: Clinical & Aperito Online Publishing & 2015 & Yes & No \\
\hline APSP Journal of Case Reports & $\begin{array}{l}\text { EL-MED-Pub Publishers/Association of Paediatric } \\
\text { Surgeons of Pakistan }\end{array}$ & 2010 & Yes & Yes \\
\hline Austin Cardio \& Cardiovascular Case Reports & Austin Publishing Group & 2015 & Yes & No \\
\hline Austin Gynecology Case Reports & Austin Publishing Group & 2015 & Yes & No \\
\hline Austin Journal of Clinical Case Reports & Austin Publishing Group & 2014 & Yes & No \\
\hline Austin Oncology Case Reports & Austin Publishing Group & 2015 & Yes & No \\
\hline Autopsy and Case Reports & Hospital Universitario of the University of San Paulo & 2011 & Yes & No \\
\hline BJR Case Reports & British Institute of Radiology & 2015 & Yes & No \\
\hline BMJ Case Reports & BMJ Publishing Group & 2008 & Optional & Yes \\
\hline Case Reports in Anesthesiology & Hindawi Publishing & 2011 & Yes & Yes \\
\hline Case Reports in Cardiology & Hindawi Publishing & 2011 & Yes & Yes \\
\hline Case Reports in Clinical Medicine & Scientific Research Publishing & 2012 & Yes & No \\
\hline Case Reports in Critical Care & Hindawi Publishing & 2011 & Yes & Yes \\
\hline Case Reports in Dermatological Medicine & Hindawi Publishing & 2011 & Yes & Yes \\
\hline Case Reports in Dermatology & Karger & 2009 & Yes & Yes \\
\hline Case Reports in Emergency Medicine & Hindawi Publishing & 2011 & Yes & Yes \\
\hline Case Reports in Endocrinology & Hindawi Publishing & 2011 & Yes & Yes \\
\hline Case Reports in Gastroenterology & Karger & 2007 & Yes & Yes \\
\hline Case Reports in Gastrointestinal Medicine & Hindawi Publishing & 2011 & Yes & Yes \\
\hline Case Reports in Genetics & Hindawi Publishing & 2011 & Yes & Yes \\
\hline Case Reports in Hematology & Hindawi Publishing & 2011 & Yes & Yes \\
\hline Case Reports in Hepatology & Hindawi Publishing & 2011 & Yes & Yes \\
\hline Case Reports in Immunology & Hindawi Publishing & 2011 & Yes & Yes \\
\hline Case Reports in Infectious Diseases & Hindawi Publishing & 2011 & Yes & Yes \\
\hline Case Reports in Internal Medicine & Sciedu Press & 2014 & Yes & No \\
\hline Case Reports in Medicine & Hindawi Publishing & 2009 & Yes & Yes \\
\hline Case Reports in Nephrology & Hindawi Publishing & 2011 & Yes & Yes \\
\hline Case Reports in Nephrology and Dialysis & Karger & 2011 & Yes & Yes \\
\hline Case Reports in Neurological Medicine & Hindawi Publishing & 2011 & Yes & Yes \\
\hline Case Reports in Neurology & Karger & 2009 & Yes & Yes \\
\hline Case Reports in Obstetrics and Gynecology & Hindawi Publishing & 2011 & Yes & Yes \\
\hline Case Reports in Oncological Medicine & Hindawi Publishing & 2011 & Yes & Yes \\
\hline Case Reports in Oncology & Karger & 2008 & Yes & Yes \\
\hline Case Reports in Ophthalmological Medicine & Hindawi Publishing & 2011 & Yes & Yes \\
\hline Case Reports in Ophthalmology & Karger & 2010 & Yes & Yes \\
\hline Case Reports in Orthopedics & Hindawi Publishing & 2011 & Yes & Yes \\
\hline Case Reports in Otolaryngology & Hindawi Publishing & 2011 & Yes & Yes \\
\hline Case Reports in Pancreatic Cancer & Mary Ann Liebert Inc. Publishing & 2015 & Yes & No \\
\hline
\end{tabular}


Table 1 Case report journals (Continued)

\begin{tabular}{|c|c|c|c|c|}
\hline Case Reports in Pathology & Hindawi Publishing & 2011 & Yes & Yes \\
\hline Case Reports in Pediatrics & Hindawi Publishing & 2011 & Yes & Yes \\
\hline Case Reports in Perinatal Medicine & De Gruyter & 2012 & Optional & No \\
\hline $\begin{array}{l}\text { Case Reports in Plastic Surgery and } \\
\text { Hand Surgery }\end{array}$ & $\begin{array}{l}\text { Taylor \& Francis/Acta Chirurgica } \\
\text { Scandinavica Society }\end{array}$ & 2014 & Yes & Yes \\
\hline Case Reports in Psychiatry & Hindawi Publishing & 2011 & Yes & Yes \\
\hline Case Reports in Pulmonology & Hindawi Publishing & 2011 & Yes & Yes \\
\hline Case Reports in Radiology & Hindawi Publishing & 2011 & Yes & Yes \\
\hline Case Reports in Rheumatology & Hindawi Publishing & 2011 & Yes & Yes \\
\hline Case Reports in Surgery & Hindawi Publishing & 2011 & Yes & Yes \\
\hline Case Reports in Transplantation & Hindawi Publishing & 2011 & Yes & Yes \\
\hline Case Reports in Urology & Hindawi Publishing & 2011 & Yes & Yes \\
\hline Case Reports in Vascular Medicine & Hindawi Publishing & 2011 & Yes & Yes \\
\hline Case Reports in Women's Health & Elsevier & 2014 & Yes & No \\
\hline Case Reports International & Edorium Journals & 2012 & Yes & No \\
\hline Case Reports: Open Access & Jscholar & 2015 & Yes & No \\
\hline Case Study and Case Report & Sageya Publishing & 2011 & Yes & No \\
\hline CEN Case Reports & Springer/Japanese Society of Nephrology & 2012 & Optional & No \\
\hline Clinical Case Reports & Wiley & 2013 & Yes & Yes \\
\hline Clinical Case Reports and Reviews & Open Access Text & 2015 & Yes & No \\
\hline $\begin{array}{l}\text { Clinical Cases in Mineral and } \\
\text { Bone Metabolism }\end{array}$ & $\begin{array}{l}\text { CIC Edizioni Internazionali/Italian Society } \\
\text { of Orthopaedics and Medicine }\end{array}$ & 2004 & Yes & Yes \\
\hline Clinical Medicine Insights: Case Reports & Libertas Academia & 2008 & Yes & Yes \\
\hline Clinics and Practice & PAGEPress & 2011 & Yes & Yes \\
\hline Cold Spring Harbor Molecular Case Studies & Cold Spring Harbor Laboratory Press & 2015 & Yes & No \\
\hline Dermatology Case Reports & OMICS International & 2015 & Yes & No \\
\hline Diabetes Case Reports & OMICS International & 2015 & Yes & No \\
\hline $\begin{array}{l}\text { Endocrinology, Diabetes, \& Metabolism } \\
\text { Case Reports }\end{array}$ & Bioscientifica & 2013 & Yes & Yes \\
\hline Epilepsy \& Behavior Case Reports & Elsevier & 2013 & Yes & Yes \\
\hline $\begin{array}{l}\text { European Journal of Case Reports in } \\
\text { Internal Medicine }\end{array}$ & European Federation of Internal Medicine & 2014 & Yes & No \\
\hline $\begin{array}{l}\text { European Journal of Pediatric } \\
\text { Surgery Reports }\end{array}$ & Thieme Medical Publishers & 2013 & Yes & Yes \\
\hline European Journal of Surgical Cases & Bilimsel Tip Yayinevi & 2010 & Yes & No \\
\hline $\begin{array}{l}\text { Experimental and Clinical Endocrinology \& } \\
\text { Diabetes Reports }\end{array}$ & Thieme Medical Publishers & 2014 & Yes & No \\
\hline $\begin{array}{l}\text { Global Journal of Medical and Clinical } \\
\text { Case Reports }\end{array}$ & PeerTechz & 2014 & Yes & No \\
\hline Grand Rounds & e-MED & 2001 & Yes & No \\
\hline Gynecologic Oncology Reports & Elsevier & 2011 & Yes & Yes \\
\hline HeartRhythm Case Reports & Elsevier/Heart Rhythm Society & 2015 & Yes & No \\
\hline Human Pathology: Case Reports & Elsevier & 2014 & yes & No \\
\hline IDCases & Elsevier & 2014 & Yes & No \\
\hline IJSS Case Reports \& Reviews & $\begin{array}{l}\text { IJSS Group of Journals/Society of Malaysian } \\
\text { Medical Association's } \\
\text { Medical Students and European Medical } \\
\text { Student's Association }\end{array}$ & 2014 & Yes & No \\
\hline Indian Journal of Medical Case Reports & CIBTech & 2012 & Yes & No \\
\hline $\begin{array}{l}\text { Interdisciplinary Neurosurgery: Advanced } \\
\text { Techniques and Case Management }\end{array}$ & Elsevier & 2014 & Yes & No \\
\hline
\end{tabular}


Table 1 Case report journals (Continued)

\begin{tabular}{|c|c|c|c|c|}
\hline $\begin{array}{l}\text { International Journal of Advances in } \\
\text { Case Reports }\end{array}$ & McMed International & 2014 & Yes & No \\
\hline $\begin{array}{l}\text { International Journal of Case Reports } \\
\text { and Images }\end{array}$ & Edorium Journals & 2010 & Yes & No \\
\hline $\begin{array}{l}\text { International Journal of Case Reports } \\
\text { in Medicine }\end{array}$ & IBIMA Publishing & 2012 & Yes & No \\
\hline International Journal of Case Studies & unclear & 2012 & Yes & No \\
\hline International Journal of Clinical Case Studies & Graphy Publications & 2014 & Yes & No \\
\hline $\begin{array}{l}\text { International Journal of Clinical Cases } \\
\text { and Investigations }\end{array}$ & unclear & 2010 & Yes & No \\
\hline $\begin{array}{l}\text { International Journal of Medical } \\
\text { and Pharmaceutical Case Reports }\end{array}$ & ScienceDomain International & 2014 & Yes & No \\
\hline International Journal of Surgery Case Reports & Elsevier & 2010 & Yes & Yes \\
\hline International Medical Case Reports Journal & Dove Medical Press & 2008 & Yes & Yes \\
\hline JAAD Case Reports & Elsevier/American Academy of Dermatology & 2015 & Yes & No \\
\hline Jacobs Journal of Clinical Case Reports & Jacobs Publishers & 2015 & Yes & No \\
\hline JBJS Case Connector & STRIATUS Orthopaedic Communications & 2011 & No & No \\
\hline JCRS Online Case Reports & $\begin{array}{l}\text { Elsevier/American Society of Cataract and } \\
\text { Refractive Surgery and European Society } \\
\text { of Cataract and Refractive Surgeons }\end{array}$ & 2013 & Yes & No \\
\hline JMM Case Reports & Microbiology Society & 2014 & Yes & No \\
\hline $\begin{array}{l}\text { Joseph Journal of Clinical Studies and } \\
\text { Medical Case Reports }\end{array}$ & Joseph Publishing Group & 2015 & Yes & No \\
\hline $\begin{array}{l}\text { Journal of Anaesthesia \& Critical Care } \\
\text { Case Reports }\end{array}$ & International Academic Research Group & 2015 & Yes & No \\
\hline Journal of Cardiology Cases & Elsevier/Japanese College of Cardiology & 2010 & No & No \\
\hline Journal of Case Reports & unclear & 2011 & Yes & No \\
\hline $\begin{array}{l}\text { Journal of Case Reports and Clinical } \\
\text { Research Studies }\end{array}$ & VRJ Publishers & 2014 & Yes & No \\
\hline $\begin{array}{l}\text { Journal of Case Reports and Images } \\
\text { in Medicine }\end{array}$ & Edorium & 2015 & Yes & No \\
\hline $\begin{array}{l}\text { Journal of Case Reports and Images } \\
\text { in Obstetrics and Gynecology }\end{array}$ & Edorium & 2015 & Yes & No \\
\hline $\begin{array}{l}\text { Journal of Case Reports and Images } \\
\text { in Oncology }\end{array}$ & Edorium & 2015 & Yes & No \\
\hline $\begin{array}{l}\text { Journal of Case Reports and Images } \\
\text { in Pathology }\end{array}$ & Edorium & 2015 & Yes & No \\
\hline $\begin{array}{l}\text { Journal of Case Reports and Images } \\
\text { in Surgery }\end{array}$ & Edorium & 2015 & Yes & No \\
\hline Journal of Case Reports and Studies & Annex Publishers & 2013 & Yes & No \\
\hline Journal of Case Reports in Medicine & Ashdin Publishing & 2012 & Yes & No \\
\hline $\begin{array}{l}\text { Journal of Case Reports in Oncology } \\
\text { and Therapy }\end{array}$ & EJourPub & 2015 & Yes & No \\
\hline Journal of Case Reports in Practice & Saman Publishing & 2013 & Yes & No \\
\hline Journal of Clinical \& Medical Case Reports & Avens Publishing Group & 2013 & Yes & No \\
\hline $\begin{array}{l}\text { Journal of Clinical and Translational } \\
\text { Endocrinology Case Reports }\end{array}$ & Elsevier & 2015 & Yes & No \\
\hline Journal of Clinical Case Reports & OMICS International & 2011 & Yes & No \\
\hline $\begin{array}{l}\text { Journal of Clinical Studies \& Medical } \\
\text { Case Reports }\end{array}$ & Herald Scholarly Open Access & 2014 & Yes & No \\
\hline Journal of Dermatological Case Reports & Specjaliści Dermatolodzy & 2007 & No & No \\
\hline $\begin{array}{l}\text { Journal of Investigative Medicine High } \\
\text { Impact Case Reports }\end{array}$ & SAGE Publications & 2013 & Yes & No \\
\hline Journal of Knee Surgery Reports & Thieme Medical Publishers & 2013 & Yes & No \\
\hline
\end{tabular}


Table 1 Case report journals (Continued)

\begin{tabular}{|c|c|c|c|c|}
\hline Journal of Medical Case Reports & BioMed Central & 2007 & Yes & Yes \\
\hline Journal of Medical Cases & Elmer Press & 2010 & Yes & No \\
\hline Journal of Neurological Surgery Reports & Thieme Medical Publishers & 2012 & Yes & Yes \\
\hline Journal of Orthopaedic Case Reports & Indian Orthopaedic Research Group & 2011 & Yes & No \\
\hline Journal of Pediatric Surgery Case Reports & Elsevier & 2013 & Yes & No \\
\hline Journal of Radiology Case Reports & EduRad Publishing & 2008 & Yes & Yes \\
\hline Journal of Surgical Case Reports & Oxford University Press & 2010 & Yes & Yes \\
\hline $\begin{array}{l}\text { Journal of Surgical Technique and } \\
\text { Case Report }\end{array}$ & Wolters Kluwer Health & 2014 & Yes & Yes \\
\hline Journal of Vascular Surgery Cases & Elsevier/Society for Vascular Surgery & 2015 & Yes & No \\
\hline JPRAS Open & $\begin{array}{l}\text { Elsevier/British Association of Plastic } \\
\text { Reconstructive and } \\
\text { Aesthetic Surgeons }\end{array}$ & 2015 & Yes & No \\
\hline JSM Clinical Case Reports & JSciMed Central & 2013 & Yes & No \\
\hline Medical Case Studies & Academic Journals & 2010 & Yes & No \\
\hline Medical Mycology Case Reports & $\begin{array}{l}\text { Elsevier/International Society for Human } \\
\text { and Animal Mycology }\end{array}$ & 2012 & Yes & Yes \\
\hline MOJ Clinical \& Medical Case Reports & MedCrave & 2015 & Yes & No \\
\hline Neurocase & Taylor \& Francis & 1995 & Optional & Yes \\
\hline NMC Case Report Journal & Japan Neurosurgical Society & 2014 & Yes & No \\
\hline OA Case Reports & OA Publishing London & 2012 & yes & No \\
\hline Oncology \& Cancer Case Reports & OMICS International & 2015 & Yes & No \\
\hline $\begin{array}{l}\text { Open Journal of Clinical and Medical Case } \\
\text { Reports }\end{array}$ & unclear & 2015 & Yes & No \\
\hline Oral and Maxillofacial Surgery Cases & Elsevier & 2015 & yes & No \\
\hline Oxford Medical Case Reports & Oxford University Press & 2014 & Yes & Yes \\
\hline Pathology Case Reviews & Wolters Kluwer Health & 1996 & No & No \\
\hline Pediatric Urology Case Reports & Hayrettin Ozturk & 2014 & Yes & No \\
\hline Radiology Case Reports & Elsevier/University of Washington & 2006 & Yes & No \\
\hline Respiratory Medicine Case Reports & Elsevier & 2008 & Yes & Yes \\
\hline Respirology Case Reports & Wiley/Asian Pacific Society of Respirology & 2013 & Yes & Yes \\
\hline Retinal Cases and Brief Reports & Wolters Kluwer Health & 2007 & Optional & Yes \\
\hline SAGE Open Medical Case Reports & SAGE Publications & 2013 & Yes & Yes \\
\hline Scholarena Journal of Case Reports & Scholarena & 2014 & Yes & No \\
\hline Scholars Journal of Medical Case Reports & SAS Publishers & 2013 & Yes & No \\
\hline $\begin{array}{l}\text { Southeast Asian Journal of Case Report } \\
\text { and Review }\end{array}$ & Sageya Publishing & 2012 & Yes & No \\
\hline Surgical Case Reports & Springer/Japan Surgical Society & 2015 & Yes & No \\
\hline $\begin{array}{l}\text { The Thoracic and Cardiovascular } \\
\text { Surgeon Reports }\end{array}$ & Thieme Medical Publishers & 2014 & Yes & Yes \\
\hline Translational Medicine Case Reports & Elsevier/European Society for Translational Medicine & 2015 & Yes & No \\
\hline Trauma Case Reports & Elsevier & 2015 & Yes & No \\
\hline Urology Case Reports & Elsevier & 2013 & yes & No \\
\hline World Journal of Clinical Cases & Baishideng Publishing Group & 2013 & Yes & Yes \\
\hline $\begin{array}{l}\text { World Journal of Medical and Surgical Case } \\
\text { Reports }\end{array}$ & Narain Publishers & 2012 & Yes & No \\
\hline
\end{tabular}




\section{Author details}

'University of Southern California Keck School of Medicine, Los Angeles County Medical Center, 12401 Washington Blvd, Whittier, CA 90602, USA. ${ }^{2} \mathrm{PIH}$ Health Hospital-Whittier Stroke Center, PIH Health Hospital Non-Invasive Vascular Laboratory, 12401 Washington Blvd, Whittier, CA 90602, USA. ${ }^{3}$ JKS Science \& Medical Writing, Los Angeles, CA, USA. ${ }^{4}$ Faculty of Medicine,

Nursing and Health Sciences, Flinders University, GPO Box 2100, Adelaide, SA 5001, Australia. ${ }^{5}$ Department of Family \& Community Medicine, University of Toronto, 500 University Avenue, Toronto M5G 1V7, Canada.

Received: 25 August 2017 Accepted: 25 August 2017

Published online: 05 October 2017

\section{Reference}

1. Rison RA, Shepphird JK, Kidd MR. How to choose the best journal for your case report. Journal of Medical Case Reports. 2017;2017:198. doi:10.1186/ s13256-017-1351-y. 